(Aus dem physiologischen Institut der Universität Wien.)

\title{
Beiträge zur Kenntnis von den Schutzeinrichtungen des Darmtractes gegen spitze Fremdkörper.
}

\author{
Von
}

Stud. med. Albert Müller.

Die folgende Untersuchung basirt auf einer Arbeit von Dr. Alfred Exner: "Wie schützt sich der Verdauungstract vor Verletzungen durch spitze Fremdkörper" ${ }^{1}$ ). Dieser fasst in Kürze die Resultate seiner Untersuchung in Folgendem zusammen: „Berührt man die Schleimhaut des Magens oder Dünndarms bei Hunden und Katzen wiederholt an derselben Stelle mit einer Nadel, so bilden sich anämische Stellen und Einziehungen der Schleimhaut am Ort der mechanischen Reizung. Diese Erscheinungen beruhen auf Contraction der Muscularis mucosae, verstärkt im Magen und Darm durch Contraction der zwischen die Drüsen aufsteigenden Muskelbündel, im Dünndarm ausserdem noch durch Contraction der Zottenmuskulatur. Bei Thieren, denen Glassplitter in den Magen und Darm gebracht wurden, finden sich diese in so gebildeten Buchten der Schleimbaut. Magen und Darm der Thiere pflegen Stecknadeln, die mit dem spitzen Ende voran eingeführt wurden, umzudrehen, so dass die Nadeln mit dem stumpfen Ende voraus, also ohne schädigende Wirkung, durch den Verdauungstract wandern."

Da sich in einigen Vorversuchen und auch bei den folgenden Versuchen stete Gelegenheit bot, die Arbeit Ex n er's zu controlliren, so möchte ich betonen, dass ich die Thatsachen derselben im wesentlichen bestätigen kann. In jedem Versuche war eine Drehung der Nadeln im Sinne der Angaben Exner's und ungefähr auch in dem von ihm angegebenen quantitativen Ausmaasse zu constatiren; niemals bemerkte ich eine Verletzung der Schleimhaut oder eine Blutung. Die Dellenbildung trat in den meisten Fällen ein, doch mit ziemlich grossen

1) Pflüger's Archiv f. d. ges. Physiologie Bd. 89 S. 253 ff. 1902. 
individuellen Schwankungen, bei manchen Thieren mit grosser Exactheit und Promptheit, bei anderen langsamer und weniger ausgeprägt. Bei zwei von meinen Versuchsthieren gelang es überhaupt nicht, Dellenbildung hervorzurufen. Auch die Narkose scheint einen Einfluss darauf auszuüben, in einigen Fällen wollten sich beim tiefnarkotisirten Thier keine Dellen erzielen lassen, die dann beim Nachlassen der Narkose prompt auftraten.

Die Frage, um deren Beantwortung ich mich bemühte, war, ob diese Erscheinungen unter dem Einflusse des Nervensystems stehen. Ich versuchte zunächst die Wirkung der Nervi vagi auf die angegebene Drehung der Nadeln durch operative Ausschaltung derselben zu studiren, und zwar verwendete ich dazu fast durchwegs Katzen, zwei Mal - mit völlig analogem Resultate - Hunde.

Die Technik der Versuche war folgende: Am narkotisirten Thiere wurden unter aseptischen Bedingungen die beiden Nervi vagi innerhalb der Bauchhöhle möglichst hoch am Durchtritt durch's Zwerchfell durchschnitten und je ein Stück des Nerven excidirt. Die Bauchhöhle wurde dann geschlossen. Die weitaus meisten Thiere uberstanden diese Operation ohue besondere Beschwerden. Der Erfolg der Operation wurde in allen Fällen durch die Obduction controllirt und bestätigt. Erst acht Tage bis drei Wochen nach der Vagotomie wurden die Thiere bei zufriedenstellendem Befinden zu den Versuchen herangezogen. Dem narkotisirten Thiere wurde nach dem Vorgang von A. Exner mit dem Schlauche eine kleine längliche Gelatinekapsel mit je 24 oder 30 Stück $13 \mathrm{~mm}$ langer Stecknadeln eingeführt und zwar so, dass je 12 resp. 15 Stück der Nadeln mit dem Kopfe und die andere Hälfte mit der Spitze voran in die Kapsel eingeschlossen wurden. Die Kapsel war selbstverständlich zu schmal, um eine Umdrehung der Nadeln in ihr zu gestatten. Bei den späteren Versuchen war die Hälfte der Nadeln durch einen Feilenstrich oder durch gelindes Plattschlagen des Kopfes gezeichnet, sodass das Schicksal der beiden Nadelgattungen getrennt verfolgt werden konnte.

Die Beobachtungen geschahen theils am toten Thiere, während sich die Nadeln noch im Darme befanden, durch directes Aufsuchen nach Eröffnung der Bauchböhle und des Darmes, meistentheils aber durch Untersuchung der Stühle.

Es braucht wohl nicht betont zu werden, dass der letzteren Art der Untersuchung verschiedene Schwierigkeiten und Un- 
vollkommenheiten anhaften. Vor Allem sind natürlich nur feste Stühle zu verwerthen, da im diarrhoischen über die Richtung der Nadeln kein Urtheil zu gewinnen ist. Ueber die Orientirung der festen Stühle entscheidet in vielen Fällen, falls keine directe Beobachtung möglich war, die charakteristische, vorne breite und abgerundete, hinten schmale und zugespitzte Form. Ferner ist auch der Umstand, dass die Nadeln, die mit dem Kopfe voran liegen, den Darmtract viel rascher zu durchwandern pflegen, mit Vorsicht zu verwerten. Die Nadeln werden nämlich von den Katzen in mehreren Stühlen im Laufe von zwei bis vier Tagen entleert, die ersten gewöhnlich nach 24 Stunden. Diese Anhaltspunkte reichen jedoch nicht in allen Fällen mit genügender Sicherheit aus, und desshalb mussten eine Anzahl von Versuchen unberücksichtigt bleiben. Auch bei den gelungenen lässt sich häufig eine geringere Anzahl von Nadeln in ihrer relativen Richtung nicht sicher angeben, so dass nicht eine bestimmte Anzahl von Nadeln als mit dem Kopfe voran und eine andere als mit der Spitze voran liegend angeführt werden können, sondern mehrere, aber gleichsinnige Combinationen möglich sind. Falls z. B. 19 Nadeln sicher mit dem Kopfe nach vorne, sieben sicher mit der Spitze voran ausgeschieden werden, und ich finde in einem Stuhlstück, über dessen Richtung ich nichts auszusagen vermag, noch vier Nadeln, drei nach der Richtung $a$, eine nach der entgegengesetzten Richtung $b$, so sind natürlich zwei Combinationen möglich: Entweder 20 Nadeln mit dem Kopfe voran $(=$ m. d. K. v. $)$ und 10 mit der Spitze voran ( - m. d. Sp. v.) oder $22 \mathrm{~m}$. d. K. v. und 8 m. d. Sp. v. - in jedem Falle ist aber eine Drebung der Nadeln in erwarteten Sinne zu behaupten.

Eine kleine Anzahl von Nadeln liegt auch desshalb unbestimmbar, weil sie quer zur Längsachse des Darmrohres gelagert ist; ein weiterer kleiner Bruchtheil entgeht auch einer sorgfältigen Untersuchung, indem er in Verlust geräth.

An den sechs Thieren (fünf Katzen und ein Hund), die die Durchschneidung der Nervi vagi völlig überstanden hatten, wurden 18 derartige Versuche angestellt, von denen ich 14 verwerten konnte. Ich möchte betonen, dass auch die nicht verwerteten Versuche in keiner Weise mit dem Resultate der gelungenen im Gegensatze zu stehen, sondern vielmehr im selben Sinne zu sprechen schienen.

Bei drei Versuchen wurden die Nadeln noch vor ihrer Entleerung am getödteten Thiere im Darme aufgesucht; beim ersten 
Thier 2 Stunden nach ihrer Einführung. Die Nadeln fanden sich - sämmtlich in unveränderter Richtung - im Magen in der Pylorusgegend, drei im obersten Dünndarm mit dem Kopfe voran. Das Resultat dieses Versuches ist in der unten folgenden Zusammenstellung nicht in Rechnung gezogen, da der Beginn der Drehung augenscheinlich noch nicht eingetreten war. Bei einem zweiten Versuche, 12 Stunden nach der Einführung von 24 Nadeln fanden sich 7 Stück im Magen $(1 \mathrm{~m}$. d. K. v. und $6 \mathrm{~m}$. d. Sp. v.), 2 Stück im Dünndarm ( $1 \mathrm{~m}$. d. K. v. und $1 \mathrm{~m}$. d. Sp. v.) und $15(1 \mathrm{~m}$. d. Sp. v. und $14 \mathrm{~m}$. d. K. v.) im Dickdarm, vorwiegend im unteren Theil des Colons, sowie im Rectum. Es zeigte sich also, dass die Nadeln m. d. K. v. den Darm raseher durchwandert hatten, und dass sich eine Drehung der Nadeln zum Kopfe in beträchtlichem Umfang eingestellt hatte $(16: 8)$. Das gleiche charakteristische Resultat ergab ein dritter derartiger Versuch mit Eröffnung der Bauchhöhle 24 Stunden nach Einführung von 30 Nadeln. 4 Nadeln m. d. K. v. hatte das Thier bereits im Stuhle entleert, im Dickdarm fanden sich 16 Nadeln $(14 \mathrm{~m}$. d. K. v. und $2 \mathrm{~m}$. d. Sp. v.), 5 Nadeln im Dünndarm $(2 \mathrm{~m}$. d. K. v. und $3 \mathrm{~m}$. d. Sp. v. $)$, 5 Nadeln im Magen (m. d. Sp. v. 4, eine quergelagert) (20:9). Von den Versuchen, wo die Beobachtung und Zählung erst im Stuhle stattfand, will ich nur die Grenzfälle herausgreifen, um die Art der Berechnung und die Schwankungen der Resultate zeigen zu können.

A. Einführung von 30 Nadeln; Entleerung derselben in drei Stühlen (I, II, III), von denen nur der erste seiner Richtung nach bestimmbar war.
M. d. K. v.
M. d. Sp. v.
Unbestimmbar
I. 13
2
1
Richtung $a \quad$ Richtung $b$
II.
6
4
1

\section{III. \\ Richtung $c$}
Je nachdem wir in II und III $a, b$ und $c$ als $\mathrm{m}$. d. K. v. oder als m. d. Sp. v. auffassen und in allen Möglichkeiten combiniren, ergeben sich als mögliche Fälle:

$\begin{array}{ccc}\text { M. d. K. v. } & \text { M. d. Sp. v. } & \text { Unbestimmbar } \\ 17 & 9 & 2 \\ 18 & 8 & 2 \\ 20 & 6 & 2\end{array}$


B. Einführung von 30 Nadeln, Entleerung in fünf Stühlen, von denen nur der erste und dritte in ihrer Richtung bestimmbar waren.

$\begin{array}{rcc} & \text { M. d. K. v. } & \text { M. d. Sp. v. } \\ \text { I. } & 11 & 4 \\ & \text { Richtung } a & \text { Richtung } b \\ \text { II. } & 4 & 3 \\ & \text { M. d. K. v. } & \text { M. d. Sp. v. } \\ \text { III. } & 1 & 1 \\ & \text { Richtung } c & \\ \text { IV. } & 3 & \\ & \text { Richtung } d & \\ \text { V. } & 1\end{array}$

Mögliche Combinationen:

$\begin{array}{cc}\text { M. d. K. v. } & \text { M. d. Sp. v. } \\ 15 & 13 \\ 16 & 12 \\ 17 & 11 \\ 18 & 10 \\ 19 & .9 \\ 20 & 8\end{array}$

Im Folgenden sollen nun die Resultate aller in Betracht kommenden Versuche zusammengefasst und ühersichtlich dargestellt werden.

Verfüttert wurden 372 Nadeln, aufgefunden davon 356 , davon in querer Richtung 13 Nadeln. Von den verbleibenden 343 Nadeln waren bestimmbar 251 , und zwar: M. d. K. v.: 198, m. d. Sp. v. 53, in unbestimmter Richtung 92.

Von diesen 92 Nadeln unbestimmter Richtung fanden sich 57 einzeln oder in Päckchen aus nur gleichgerichteten Nadeln, so dass man sie gleicbmässig für jede Richtung in Anspruch nehmen könnte, die übrigen 35 befanden sich in versehiedener Anzahl immer mit auch entgegengesetzt gerichteten Nadeln zusammen. Diese lassen sich in verschiedener Weise gruppiren; wenn 26 nach der Richtung $a$ liegen, so müssen 9 die Richtung $b$ einnehmen. Das ist der extreme Fall, doch sind auch geringere Differenzen zwischen $a$ und $b$ möglich. Nehmen wir nun den für die Drehung ungüustigsten Fall, d. $\mathrm{h}$. betrachten wir die 26 Nadeln als mit der Spitze voran liegend, so gelangen wir zu folgendem Verbältniss: 


\begin{tabular}{cc} 
M. d. K. v. & M. d. Sp. v. \\
198 & 53 \\
9 & 26 \\
\hline 207 & 79
\end{tabular}

Der für die Drehung günstigste Fall ist:

\begin{tabular}{cc} 
M. d. K. v. & M. d. Sp. v. \\
198 & 53 \\
26 & 9 \\
\hline 224 & 62
\end{tabular}

Das Mittel beträgt:

$$
\begin{aligned}
& \text { M. d. K. v. M. d. Sp. v. } \\
& 215: 71=3: 1 \text {. }
\end{aligned}
$$

Theilen wir diesem Verhältniss entsprechend die 57 einzeln gelegenen Nadeln, so kommen wir zu dem Wahrscheinlichkeitsresultat:

$$
\begin{array}{cl}
\text { M. d. K. v. } & \text { M. d. Sp. v. } \\
253(215+38) & 90(71+19)
\end{array}
$$

Aber selbst bei dem denkbar ungünstigsten Fall, wenn wir alle 57 Nadeln als m. d. Sp. v. liegend ansehen, bleibt noch ein ansehnlicher Ueberschuss von Nadeln, die m. d. K. v. den Darm verlassen, nämlich :

$$
\begin{array}{cc}
\text { M. d. K. v. } & \text { M. d. Sp. v. } \\
207(198+9) & 136(53+57+26)
\end{array}
$$

Aus dem Gesagten geht eindeutig hervor, dass auch nach Vagusdurchschneidung eine grössere Anzahl von Nadeln gedreht werde und zwar so gedreht, dass sie mit dem Kopfe voran zu liegen kommen, d. h. unschädlich werden. Nach den angeführten Zahlen dürfte ein Zufall wohl auszuschliessen sein. Den Nervi vagi scheint also ein Einfluss auf die Drebung von spitzen Fremdkörpern im Verdauungstracte nicht zuzukommen.

Ich habe schon oben erwähnt, dass die späteren Versuche mit nach der Einführungsrichtung bezeichneten Nadeln ausgeführt wurden. Der gegen diese Methodik mögliche Einwand, dass die Gelatinekapsel mit den Nadeln sich leicht vor ihrer Auflösung als Ganzes im Magen hätte drehen können und so die Einführungsverhältnisse sich geändert hätten, erledigt sich am leichtesten durch die Thatsache, dass dies augenfällig bei meinen Versuchen, vielleicht zufällig, nicht der Fall war; aber auch wenn das nicht so gewesen wäre, hätte das raschere Erscheinen der angeblich mit der Spitze voran eingeführten 
Nadeln, sowie die Aenderung der zu schildernden Drehungsverhältnisse die Drehung der Kapsel im Magen erschliessen lassen.

Diese Versuche sicherten die Beobachtung, dass die m. d. K. v. eingeführten Nadeln den Darmtract weit rascher durchwandern und ergaben übereinstimmend das überraschende Nebenresultat, dass auch die mit dem Kopfe voran eingeführten Nadeln theilweise in die entgegengesetzte Richtung gedreht werden und so den Darm verlassen können. Man darf also nicht den Satz aufstellen, dass die Nadeln in die Lage m. d. K. v. gedreht werden, sondern nur, dass die Mehrzahl derselben, vielleicht erst nach wiederholten Drehungen, in die genannte Stellung gelangt. Da diese Versuche mit gezeichneten Nadeln untereinander übereinstimmen, will ich nur das Resultat eines herausgreifen.

Eine vagotomirte Katze wurde mit 30 bezeichneten Nadeln gefüttert, die in 4 Stühlen ausgeschieden wurden. Ich will in der folgenden Tabelle zur Anzabl derer, welche m. d. K. v. eingeführt wurden, ein „ $k^{\prime}$, zu denjenigen, welche $\mathrm{m}$. d. Sp. v. eingeführt wurden, ein "s" hinzufügen.

M. d. K. v.

I. $\quad 2(2 k)$

II. $5(4 k, 1 s)$

III. $\quad 14(5 k, 9 s)$

Richtung $a$

IV. $\quad 3(1 k, 2 s)$
M. d. Sp. v.

$3(2 k, 1 s)$

Richtung $b$

$2(2 s)$

Es wurden also von 29 aufgefundenen Nadeln 24 resp. 23 m. d. K. v. ausgeschieden, zunächst durchaus Nadeln m. d. K. v., und zwar am raschesten diejenigen, die auch m.d. K.v. eingeführt waren, aber 2 resp. 3 Nadeln, die m. d. K. v. eingeführt waren, wurden m. d. Sp. v. entleert.

Völlig analog sind die Verhältnisse nach möglichster Ausschaltung des sympathischen Nervensystems, nämlich nach Exstirpation des Plexus solaris. Es wurden 6 Thiere (5 Katzen, 1 Hund) operirt und am Leben gelassen. Drei davon überstanden die Operation, drei gingen zu Grunde, u. z. eines an eitriger Peritonitis, zwei aus unbekannter Ursache, vielleicht Shokwirkung, wie dies nach Exstirpation der Ganglia coeliaca schon vielfach von anderer Seite beobachtet wurde. Die übrigen Thiere konnten verwendet werden, eines starb 14 Tage nach der Operation aus unbekannter Ursache, eines 3 Wochen nachher an einer interkurrenten Erkrankung, eines 
ging nach $1^{1 / 2}$ Monaten an dem Versuch zu Grunde, zu der Ausschaltung des sympathischen Nervensystems auch die Durchschneidung der Nervi vagi hinzuzufügen, keines aber während eines Versuches oder an den unmittelbaren Folgen desselben.

Ausser einer beträchtlichen Abmagerung, einer Abnahme der Lebhaftigkeit und Fresslust der Thiere habe ich keine auffallende Veränderung an ihnen bemerkt. Insbesondere war der Stuhl völlig normal, d. h. gewöhnlich geformt und ohne makro- oder mikroskopische Beimengung von Blut. Aufgefallen ist mir, dass die Wanderung der Nadeln durch den Darmtract nach Exstirpation der Ganglia coeliaca mehr Zeit zu erfordern schien, nämlich 5-6 Tage, und selbst erst nach 7-8 Tagen fand ich die letzten Nadeln.

An diesen Thieren wurden 7 Versuche angestellt, von denen ich 6 verwerthen konnte. Da die Ausführung and Verwerthung der Versuche, denen nach Vagusdurchschneidung in allen Einzelheiten gleicht, will ich nur einen Versuch ganz, und dann das Gesammtresultat der übrigen anführen.

Es wurden 24 bezeichnete Nadeln, je $12 \mathrm{~m}$. d. Sp. v. und $12 \mathrm{~m}$. d. K. v. eingeführt und 23 aufgefunden und zwar:

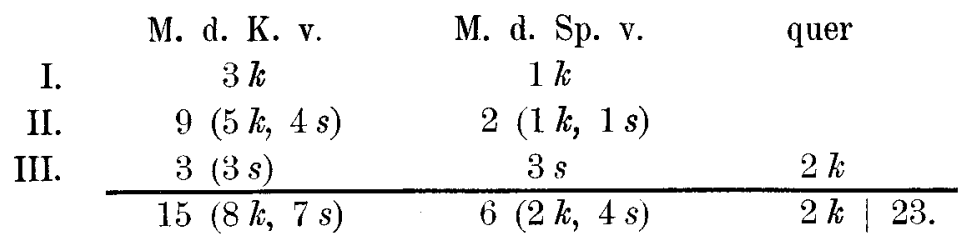

Insgesammt wurden 168 Nadeln verfüttert, 157 aufgefunden, davon quer 4 , es verbleiben 153 , davon m.d.K. v.: 91, m.d.Sp. v.: 36 , in unbestimmter Richtung: 26 , davon einzeln oder in nur gleichgerichteten Päckchen: 15, zusammen mit auch entgegengesetzt gerichteten Nadeln: 11 und zwar in zwei Päckchen: 6 und 2, 2 und 1, so dass zwei Combinationen möglich sind: Richtung $a: 8$ oder 7 , Richtung $b: 3$ oder 4 .

Der für die Drehung ungünstigste Fall ist:

$$
\begin{aligned}
& \text { M. d. K. v.: } 94(91+3) \\
& \text { M. d. Sp. v.: } 59(36+15+8) \text {, }
\end{aligned}
$$

der für die Drehung günstigste:

$$
\begin{aligned}
& \text { M. d. K. v.: } 114(91+15+8) \\
& \text { M. d. Sp. v.: } \quad 39(36+3) .
\end{aligned}
$$


Das Mittel beträgt: M. d. K. v.: 104 , m. d. Sp. v.: 49, also ca. $2: 1$. Die Erscheinungen, dass die Nadeln mit dem Kopfe voran den Darm rascher durcheilten, dass auch Drehungen von m. d. K. v. eingeführten Nadeln erfolgten, wiederholten sich.

Hervorheben möchte ich noch, dass in keinem Falle, weder nach Vagusdurchschneidung noch nach Exstirpation des Plexus solaris sich eine Verletzung oder Blutung im Magen oder Darme finden liess, dass keine Nadel sich in die Schleimhaut eingebohrt oder sie gar durchbohrt hatte.

Die merkwürdige Erscheinung, dass spitze Fremdkörper den Darmtract durchwandern ohne zu verletzen, sowie dass ein beträchtlicher Theil von ihnen so gedreht wird, dass er unschädlich ist (dass Nadeln mit dem Kopfe voraus zu liegen kommen), hat also auch für den theilweise entnervten Darm Geltung. Der Ausdruck "entnervter Darm" ist allerdings nur bedingt richtig. bleiben ja doch die Ganglien und Nervengeflechte der Darmwand selbst intact. Auch ist ein Mitspielen der directen Erregbarkeit der Darmmuskulatur nicht ausgeschlossen. Eine Scheidung der Function einerseits der im Darmrohr enthaltenen nervösen, andererseits der muskulösen Organe auf experimentellem Wege liess sich für unsere Frage nicht erbringen, da die Ausschaltung der nervösen Elemente der Darmwand durch Nikotinisirung für den ganzen Darm und für die Dauer eines Versuches vollkommen ausgeschlossen ist.

Die zweite Erscheinung, die Exner beschrieben hat, ist die Dellenbildung im Darme bei Reizung mit einer Spitze. Wo Falten vorhanden sind, z. B. im Magen, lassen sich an ihnen Veränderungen hervorbringen, kleinere Falten verschwinden völlig. Die Reizung geschieht am besten durch ziemlich energisches Streichen und Sticheln mit einem Unterbindungshaken oder einer stumpfen Nadel. Es lässt sich dabei das mechanische Moment nicht völlig ausschliessen, doch ist diese Erscheinung rein mechanisch nicht zu erklären, wie Exner schon hervorgehoben hat.

Es lag nun nàhe, den Einfluss des Nervensystems auch auf diese Erscheinung zu studiren und zwar zunächst den der Nervi vagi. Das geschah nun auch in 11 Versuchen. Es wurden dazu theils die vagotomirten Thiere verwendet, in der grösseren Anzahl jedoch ein anderer Weg gewählt. Es wurde am normalen narkotisirten Thiere zunächst das Verschwinden der Falten im Magen, gewöhnlich auch 
im Darme geprüft, dann vagotomirt und wieder untersucht und verglichen. Bei dieser Gelegenheit stellten sich individuelle Verschiedenheiten, sowie das Ausbleiben der Reaction in zwei Fällen heraus.

Ich will nun gleich hervorheben, dass es nicht gelang, in dieser Frage zu einem eindeutigen Resultat zu gelangen. Die ersten sechs Versuche machten mich glauben, dass nach der Durchschneidung der Nervi vagi die in Rede istehende Reaction im ganzen Darmtract, speciell auch im Magen, völlig unterbleibe, ein gewiss merkwürdiges und auffallendes Resultat. Ich füge eines meiner Versuchsprotokolle, das vom 16. Januar 1903, an.

„An der narkotisirten Katze wurde der Magen eröffnet. An ihm liessen sich die Hauptfalten nicht zum Verstreichen bringen, dagegen Uebergangsfalten unterbrechen und Grübchen bilden. Nach Durchschneidung der Nervi vagi in der Bauchhöhle liessen sich Falten auf keine Weise unterbrechen oder Grübchen erzielen. Die Schleimhaut zeigte ein eigentbümlich todtes und reactionsloses Verhalten; die Falten liessen sich nur passiv wie bei einem Stück Stoff verschieben, aber veränderten sich nicht.

Nur einmal schien ganz an der Schnittfläche eine Spalte zu verstreichen, wobei jedoch passiver Zug nicht ausgeschlossen werden konnte. Der Unterschied war unverkennbar. Am Dünndarm liessen sich durch Striche mit der Nadel einige Grübchen erzielen, doch ergab eine Stelle, die der kalten Luft ausgesetzt wurde, ganz ähnliche Bildungen. Diese Grübchen konnten auch durch Anspannen der Darmwand zum Verstreichen gebracht werden."

Im Gegensatz hierzu ein anderes Versuchsprotokoll vom 6. Februar 1903: „Ziemlich prompte Reaction vor Durchschneidung der Vagi, nach Durchschneidung schien die Reaction sich im Allgemeinen träger und langsamer einzustellen, doch liessen sich einzelne Falten mit Sicherheit unterbrechen, auch bildeten sich Grübchen".

In einem Falle schien die Reaction vor und nach Durchschneidung der Vagi kaum zu differiren.

An Anomalien des Nervenverlaufes in diesen Fällen liess sich schon desshalb nicht denken, weil in einigen die anatomisch-unzweideutigen Vagi am Halse oder in der Brusthöhle durchschnitten wurden. Der Obductionsbefund wies in allen Fällen eine vollständige Durchschneidung der Nervi vagi auf.

Auch wäre es nach meinem subjectiven Eindruck kaum thunlich, den oft eclatanten Unterschied nach Vagusdurchschneidung auf die 
Aenderung der Bedingungen, d. $h$. die längere Dauer des Versuches und die Narkose, den Zutritt der Luft zur Bauchhöhle und den operativen Eingriff als solchen zurückzuführen.

Ich theile diese sich theilweise widersprechenden Ergebnisse tale, quale mit, ohne dass ich eine bestimmte Meinung darüber äussern möchte.

Bei Exstirpation der Ganglia coeliaca war ein Unterschied der Reaction vor und nach der Operation nicht zu bemerken, ebenso wenig nach Bestreichen einzelner Theile des Darmes und Magens mit Nikotinlösung zur Ausschaltung der Nervenelemente des Darmes.

Anhangsweise sei noch Folgendes erwähnt: Auch Nadeln, welche $25 \mathrm{~mm}$ lang waren, also in Darm der Katze nicht gedreht werden konnten, passirten den Darmtract, ohne Schaden oder Verjetzungen anzurichten; diese Nadeln wurden in einem durch festere Kothmassen, Strohhalme und Haare gebildeten Packetchen aufgefunden, hatten ihre Richtung nicht geändert und waren beieinander geblieben. Dabei sei hervorgehoben, dass der contrahirte Katzendünndarm ein offenes Lumen nicht zeigt, der gut gefüllte einen grössten Durchmesser von 12 bis $14 \mathrm{~mm}$ aufweist, $2 \mathrm{~cm}$ im Durehmesser aber niemals erreicht oder überschreitet.

Als Resultat der Untersuchung fasse ich zusammen:

1. Die von A. Exner beschriebene Umdrehung der Nadeln erfolgt auch nach Ausschaltung der Nervi vagi und des Plexus solaris, muss also auf eiren Mechanismus bezogen werden, der im Darm selbst liegt.

2. Auch nach dieser Ausschaltung durchwandern spitze Fremdkörper den Darm, ohne ihn zu durchbohren oder zu verletzen.

3. Die Umdrehung der Nadeln erfolgt nicht nur in dem Sinne, dass Nadeln, die mit der Spitze voran liegen, in die Richtung mit dem Kopfe voran gebracht werden, sondern es findet regelmässig, nur in geringerem Umfang auch der entgegengesetzte Vorgang statt.

4. Ein Einfluss der Innervation auf die Erscheinungen der Dellenbildung und des Verstreichens der Falten lässt sich nicht mit Sicherheit nachweisen.

Zum Schlusse sei es mir gestattet, Herrn Hofrath Prof. S. Exner, von dem die Anregung zur vorliegenden Arbeit ausging, und Herrn Prof. Dr. A. Kreidl, unter dessen Leitung die Ausführung geschah, meinen wärmsten Dank auszusprechen. 